\title{
Developmental Timing of High Fat Diet Exposure Impacts Glucose Homeostasis in Mice in a Sex-Specific Manner
}

Maria M. Glavas ${ }^{1}$, Ann Y. Lee ${ }^{1}$, Ian Miao ${ }^{1}$, Fan Yang ${ }^{1}$, Majid Mojibian ${ }^{1}$, Shannon M. O’Dwyer ${ }^{1}$, Timothy J. Kieffer ${ }^{1,2,3}$

ONLINE SUPPLEMENTARY MATERIALS

\section{MATERIALS}

CFSE Proliferation Assay. $20 \times 10^{6}$ splenocytes incubated in Hanks buffer containing 20 mM HEPES without serum (Life Technologies, Carlsbad, CA) were labeled with $2 \mu \mathrm{M}$ 5,6-carboxyfluorescein diacetate, succinimidyl ester (Molecular Probes, Eugene, OR). Cells were then incubated for 20 min at $37^{\circ} \mathrm{C}$ with gentle shaking and then washed with cold HBSS medium containing $20 \mathrm{mM}$ HEPES and 5\% heatinactivated FBS. Labelled cells were resuspended in RPMI-1640 containing 5\% heat-inactivated FBS, 2 mM L-glutamine, $25 \mathrm{mM}$ HEPES, $50 \mu \mathrm{M} \beta$-mercaptoethanol and $1 \%$ antibiotic/antimycotic to a final concentration of $1.2 \times 10^{6}$ cells/well in a 24 -well plate. Insulin peptide $(10 \mu \mathrm{g} / \mathrm{mL})$, ovalbumin $(1 \mu \mathrm{M})$, or Concavalin A (ConA; $5 \mu \mathrm{g} / \mathrm{mL}$ final concentration) were added to each well and plates were incubated at $37^{\circ} \mathrm{C}$ in $5 \% \mathrm{CO}_{2}$. After 3 days, rhlL-2 was added to each well to a final concentration of $10 \mathrm{U} / \mathrm{mL}$ and plates were incubated until day eight. Cell proliferation was assessed using CFSE-based flow cytometry.

Cyclophosphamide Study. Male Swiss Webster mice (4 months old), maintained on NIH-31M diet since birth, were injected intraperitoneally with $200 \mathrm{mg} / \mathrm{kg}$ cyclophosphamide (Sigma-Aldrich, St. Louis, MO). A second $200 \mathrm{mg} / \mathrm{kg}$ cyclophosphamide injection was administered 50 days later. Mice were monitored for development of diabetes by weekly monitoring of $4 \mathrm{hr}$ fasted blood glucose. 
Supplementary Table 1. Immune cell infiltration and white adipose tissue gross abnormalities in aged (postnatal day 325) females.

\begin{tabular}{lcccc}
$\begin{array}{c}\text { Treatment } \\
\text { Group }\end{array}$ & $\begin{array}{c}\text { \% exhibiting } \\
\text { WAT gross } \\
\text { abnormalities }\end{array}$ & $\begin{array}{c}\text { \% mice exhibiting } \\
\text { liver immune cell } \\
\text { accumulation }\end{array}$ & $\begin{array}{c}\text { \% mice exhibiting } \\
\text { peri-insulitis/insulitis }\end{array}$ & $\mathbf{n}$ \\
\hline CHOW & 0 & 50 & 67 & 6 \\
HFD $^{\text {P2-21 }}$ & 33 & 0 & 33 & 6 \\
HFD $^{\text {P21-35 }}$ & 33 & 100 & 83 & 6 \\
HFD $^{\text {P2-35 }}$ & 40 & 60 & 100 & 5 \\
HFD $^{\text {P35+ }}$ & 33 & 0 & 0 & 3 \\
HFD $^{\text {P21+ }}$ & 100 & 33 & 50 & 6 \\
HFD $^{\text {P2-21,P35+ }}$ & 100 & 67 & 67 & 3 \\
HFD $^{\text {P2+ }}$ & 80 & 60 & 80 & 5 \\
\hline
\end{tabular}

White adipose tissue (WAT) gross abnormalities were defined by yellow colouring of perigonadal and/or mesenteric WAT identified at necropsy. Liver immune cell accumulation was defined as any observation of a cluster of immune cells adjacent to portal vein or central vein in an H\&E liver section. Peri-insulitis/insulitis was defined as any observation of immune cells clustered directly next to an islet or infiltrating an islet. Superscript in group name indicates postnatal days (P) of high-fat diet (HFD) exposure, with CHOW exposure at other days. 

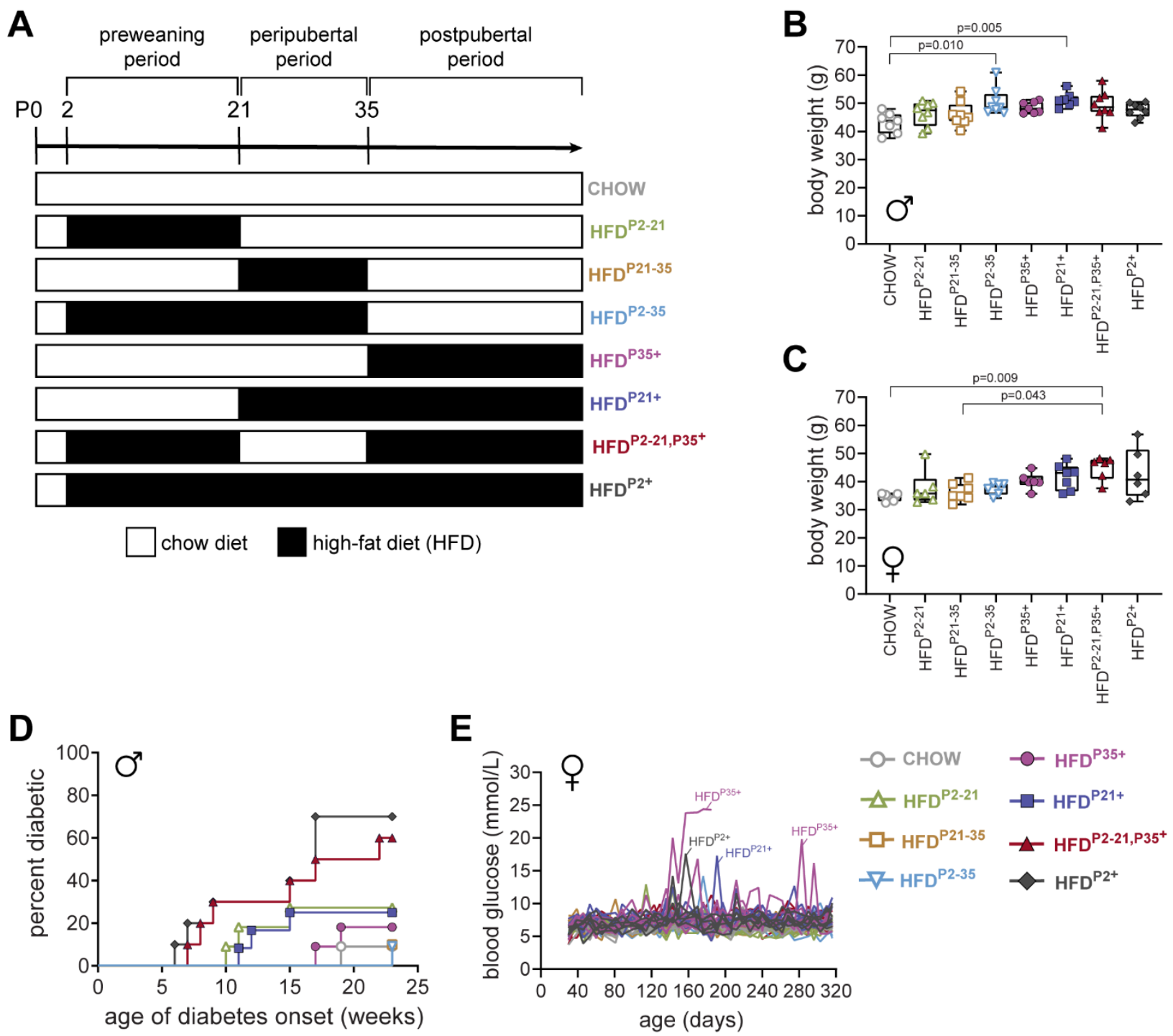

Supplementary Figure 1. (A) Schematic of diet treatment groups for mice studied into adulthood. Dams were maintained on chow diet throughout pregnancy and either remained on this diet throughout the lactation (preweaning) period or were switched to HFD diet. Pups were weaned at P21 and either kept on the same diet as their mothers or diet was switched. The final diet switch of offspring was performed at P35. Body weights of (B) male and (C) female offspring at P66. (D) Diabetes incidence in male offspring followed to 23 weeks of age, $n=10-11$ / group. Day of diabetes onset was defined as the first of two consecutive fasting blood glucose levels $>20 \mathrm{mmol} / \mathrm{L}$. (E) Fasting blood glucose tracking in females (each line represents an individual mouse) from P30 to 316, $n=6-7$ / group. One HFD $^{\text {P35+ }}$ female developed diabetes and was euthanized. Additional labeled traces indicate mice that had transient elevations in blood glucose that approached diabetic levels but returned to normal (one HFD ${ }^{\mathrm{P} 35+}$, one $\mathrm{HFD}^{\mathrm{P} 21+}$, one $\mathrm{HFD}^{\mathrm{P} 2+}$ ). 


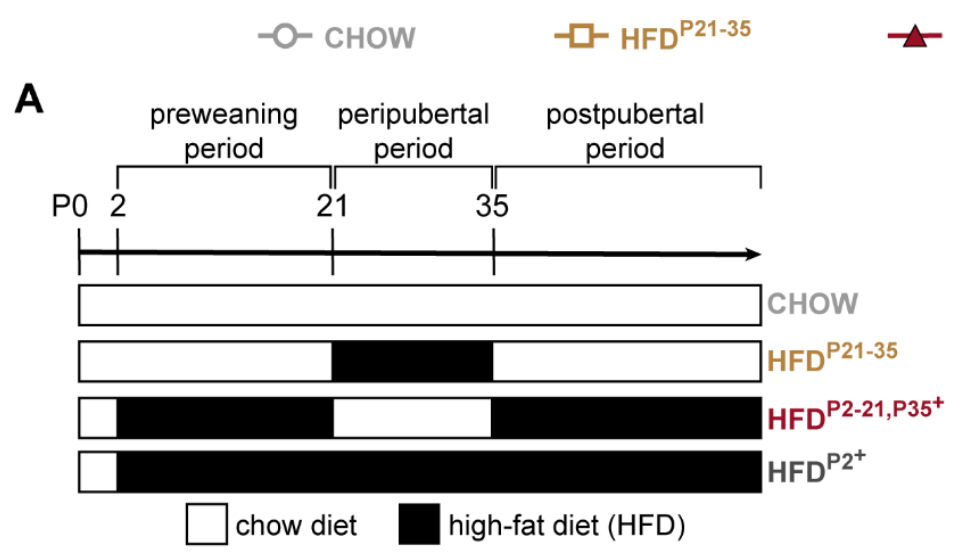

$\mathrm{HFD}^{\mathrm{P} 2-21, \mathrm{P}^{+}} \quad \prec \mathrm{HFD}^{\mathrm{P} 2^{+}}$

A

B

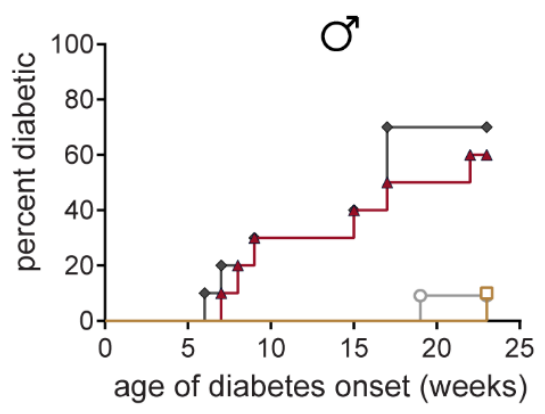

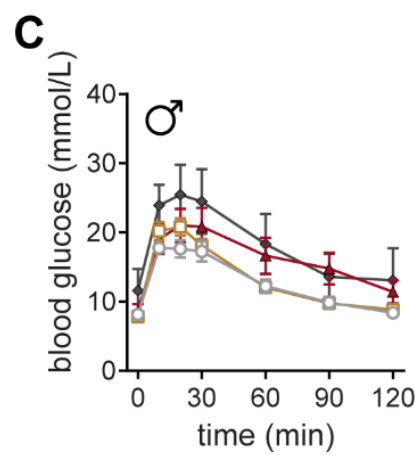

$\mathbf{E}$

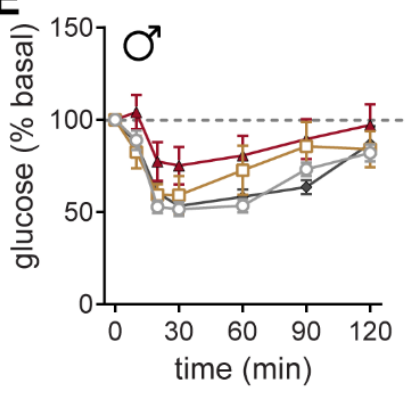

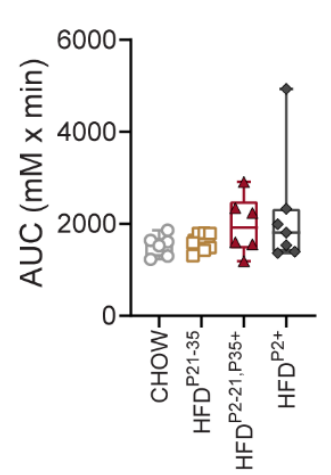

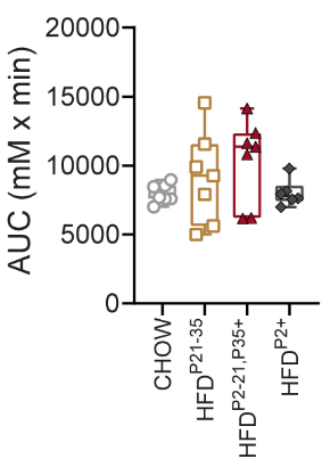

D
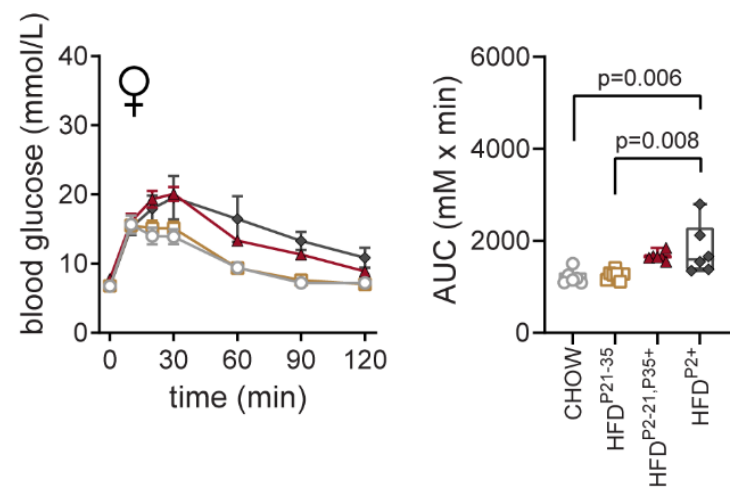

$\mathbf{F}$

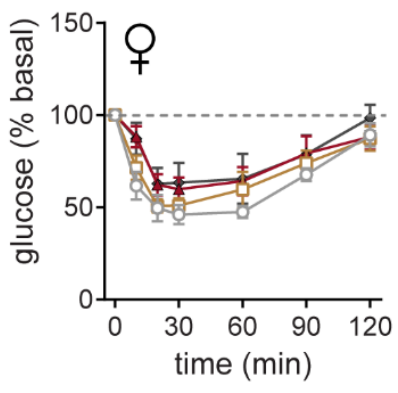

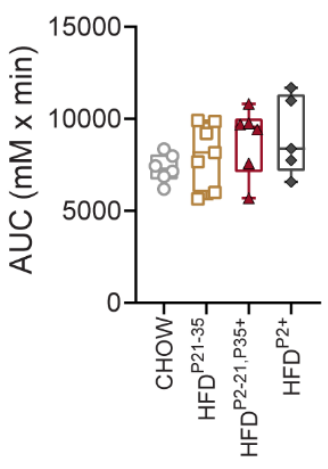

Supplementary Figure 2. Short-term diet switch during peripuberty has minimal effect on glucose homeostasis. (A) Schematic of diet treatment groups. Dams were maintained on chow diet (designated by white bars) through pregnancy and until pup postnatal day (P) 2, at which some dams were placed on high fat diet (HFD; designated by black bars). Pups were weaned at P21, at which time they were either kept on the same diet as dams or diet was switched as indicated. Some offspring had an additional diet switch at P35, as indicated. (B) Diabetes incidence to 23 weeks of age in males, $n=10-11$ / group. HFD ${ }^{\mathrm{P2}+}$ $>\mathrm{CHOW}$ and HFD ${ }^{\mathrm{P} 21-35}, \mathrm{p}^{\prime} \mathrm{s}<0.005 ; \mathrm{HFD}^{\mathrm{P2}-21, \mathrm{P}^{35+}}>\mathrm{HFD}^{\mathrm{P21}-35}$ and $\mathrm{CHOW}, \mathrm{p}^{\prime} \mathrm{s}<0.05$ by log-rank tests. Glucose response and area under the curve (AUC) during oral glucose tolerance test in (C) males at P50 and (D) females at P88, and glucose response and AUC during insulin tolerance test in $(E)$ males and $(F)$ females at P66. Note that data from $\mathrm{CHOW}$ and $\mathrm{HFD}^{\mathrm{P2}+}$ control groups are the same in Figures 2, 3, and Supplementary Figure 2, with all statistical analyses controlling for multiple comparisons of all 8 diet groups. 
A

Grade 0

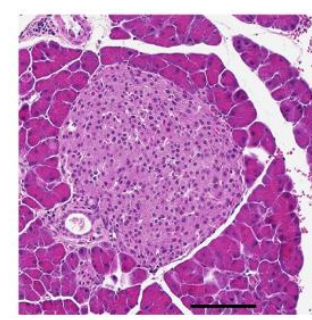

Grade 1

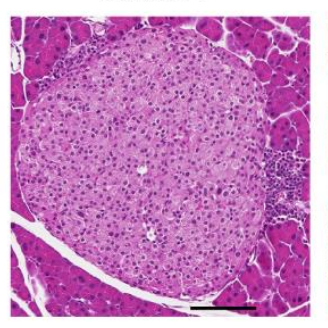

Grade 2

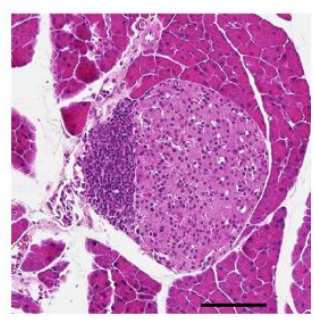

Grade 3

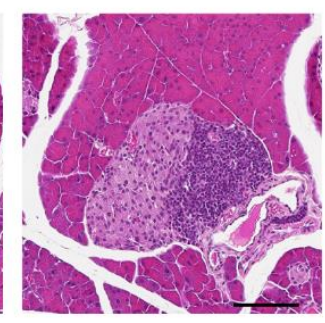

Grade 4

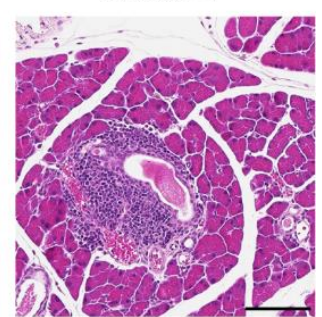

B

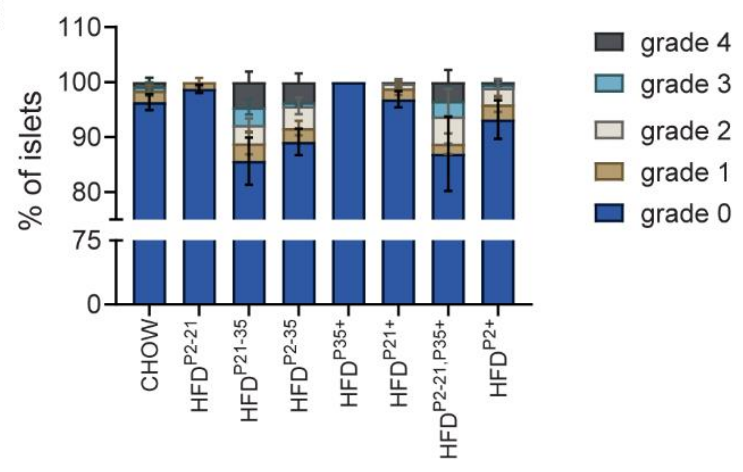

Supplementary Figure 3. Insulitis grading. (A) Representative H\&E stained islets of insulitis grading scale. Grade 0: no visible immune cell infiltration; Grade 1: mild peri-insulitis; Grade 2: moderate peri-insulitis, some infiltration; Grade 3: $\geq 50 \%$ of islet infiltrated; Grade 4: complete or near complete islet infiltration. Scale bars $=100 \mu \mathrm{m}$. (B) One pancreas section per female Swiss Webster mouse at postnatal day 325 was assessed, with each islet assigned a grade. Data represent the mean \pm SEM of the percent of islets within each grade per section ( $\mathrm{n}=6$ for $\mathrm{CHOW}, \mathrm{HFD}^{\mathrm{P2}-21}$, HFD ${ }^{\mathrm{P21}-35}$, and $\mathrm{HFD}^{\mathrm{P} 21+} ; \mathrm{n}=5$ for $\mathrm{HFD}^{\mathrm{P2}-35}$ and $\mathrm{HFD}^{\mathrm{P2}+} ; \mathrm{n}=3$ for HFD ${ }^{\mathrm{P} 35+}$ and $\mathrm{HFD}^{\mathrm{P2}-21,35+}$ ). 

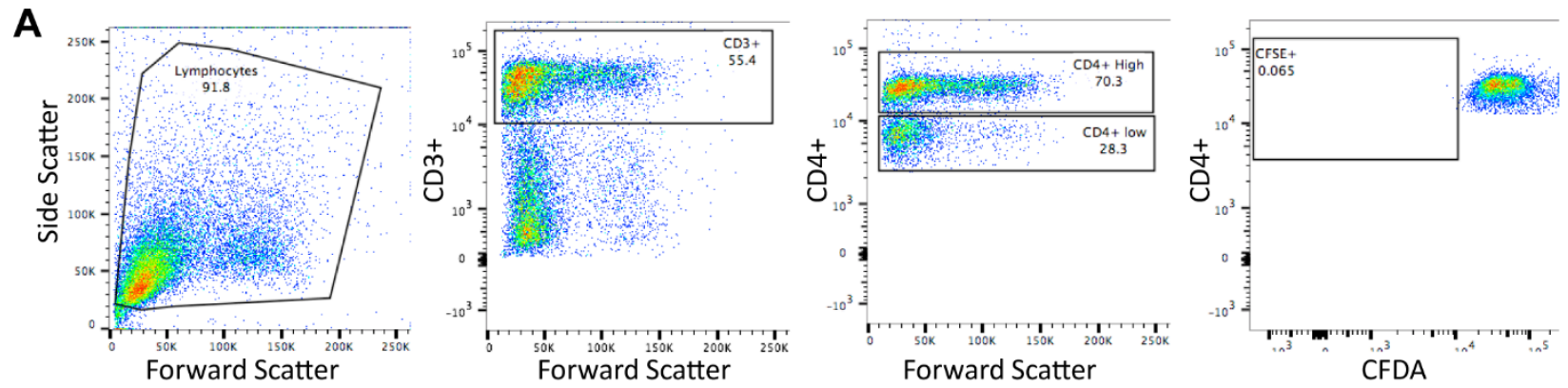

B
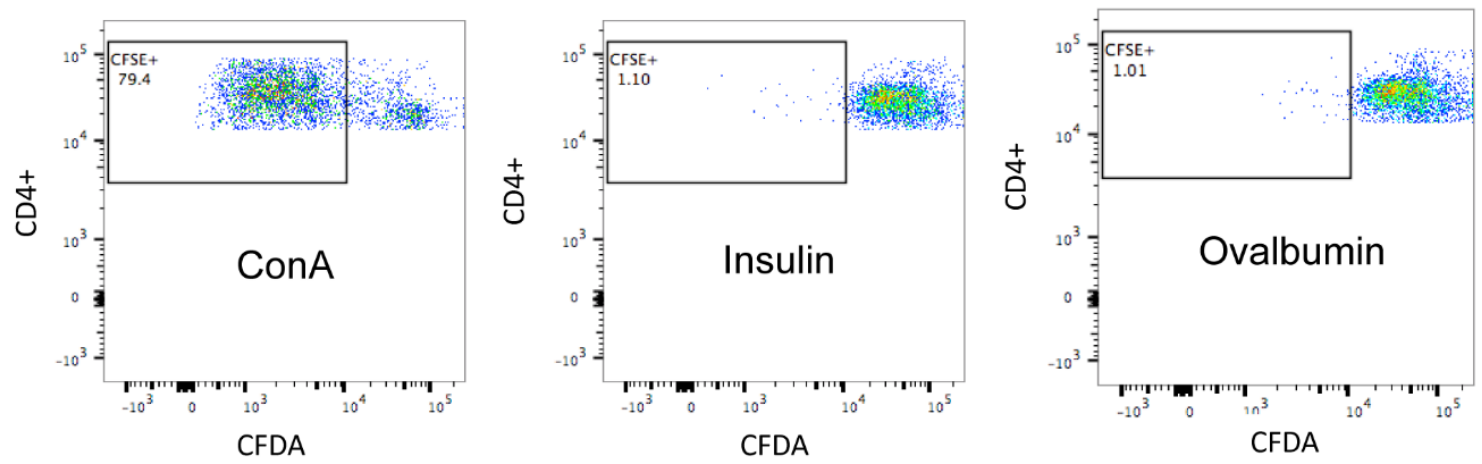

C

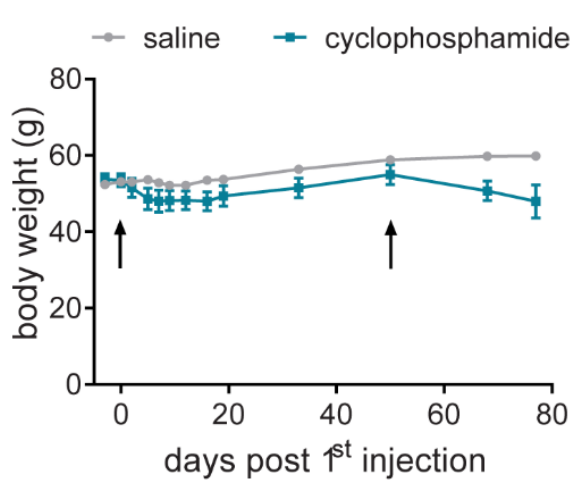

D

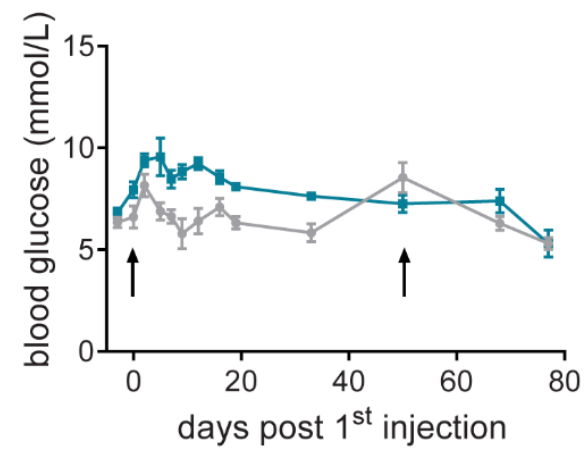

Supplementary Figure 4. Swiss Webster male mice are not prone to development of autoimmune diabetes. (A) Splenocytes were labeled with carboxyfluorescein succinimidyl ester (CFSE) and incubated with (B) ConA, insulin, or ovalbumin. On day 3 of culture rhIL-2 was added to each well. Cell proliferation was assessed using a CFSE-based flow cytometric assay. (C,D) Cyclophosphamide $(200 \mathrm{mg} / \mathrm{kg})$ was administered intraperitoneally at day 0 and 50 , indicated by arrows, and body weight and blood glucose monitored to day 78 to assess induction of autoimmune diabetes. 